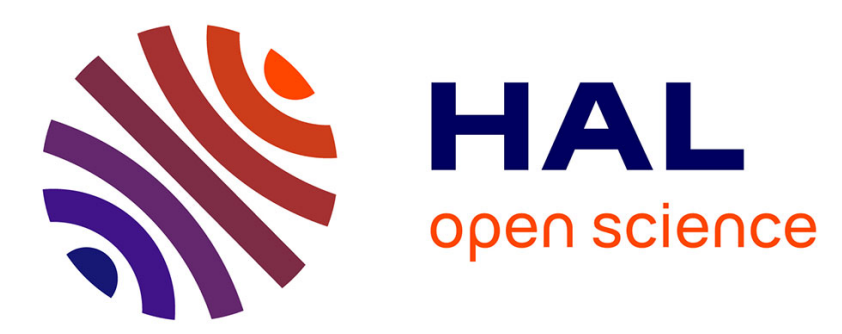

\title{
Régulation précise et automatisation d'une installation de pression pour viscosimétrie capillaire
}

\author{
M. N. Raphalen, C. Wolff
}

\section{To cite this version:}

M. N. Raphalen, C. Wolff. Régulation précise et automatisation d'une installation de pression pour viscosimétrie capillaire. Revue de Physique Appliquée, 1972, 7 (4), pp.353-356. 10.1051/rphysap:0197200704035300 . jpa-00243638

\section{HAL Id: jpa-00243638 https://hal.science/jpa-00243638}

Submitted on 1 Jan 1972

HAL is a multi-disciplinary open access archive for the deposit and dissemination of scientific research documents, whether they are published or not. The documents may come from teaching and research institutions in France or abroad, or from public or private research centers.
L'archive ouverte pluridisciplinaire HAL, est destinée au dépôt et à la diffusion de documents scientifiques de niveau recherche, publiés ou non, émanant des établissements d'enseignement et de recherche français ou étrangers, des laboratoires publics ou privés. 
Classification

Physics Abstracts

14.70

\title{
RÉGULATION PRÉCISE ET AUTOMATISATION D'UNE INSTALLATION DE PRESSION POUR VISCOSIMÉTRIE CAPILLAIRE
}

\author{
M. N. RAPHALEN et C. WOLFF \\ Laboratoire d'Hydrodynamique Moléculaire, Université de Bretagne Occidentale, \\ 6 , avenue V. Le Gorgeu, 29N-Brest.
}

(Reçu le 29 octobre, révisé le 23 mai 1972)

\begin{abstract}
Résumé. - Description d'une régulation de pressions comprises entre 10 et $1200 \mathrm{mb}$ adaptable à des viscosimètres capillaires. Les 16 valeurs possibles de la pression, régulées à $10^{-2} \mathrm{mb}$ près jusqu'à $270 \mathrm{mb}$ et à $10^{-1} \mathrm{mb}$ au-delà, permettent d'effectuer automatiquement des cycles de mesures viscosimétriques aux 16 contraintes de cisaillement correspondantes. Les mesures effectuées avec du toluène ont montré une très bonne reproductibilité des temps d'écoulement et une précision relative supérieure à $2 \times 10^{-4}$.
\end{abstract}

\begin{abstract}
A regulator for pressures between 10 and $1200 \mathrm{mb}$, for capillary viscometry is described. 16 values of the pressure, with an accuracy of $10^{-2} \mathrm{mb}$ up to $270 \mathrm{mb}$ and $10^{-1}$ at higher pressure, allows one to perform automatically measurements over the 16 corresponding values of the shear stress. Measurements on toluol show a very good reproductibility on flow times and a relative precision better than $2 \times 10^{-4}$.
\end{abstract}

Introduction. - Dans l'étude viscosimétrique des solutions ou des suspensions diluées (par exemple de macromolécules) il est souvent utile de déterminer la viscosité intrinsèque.

$$
[\eta]=\lim _{C \rightarrow 0} \frac{\left(\eta-\eta_{0}\right)}{\eta_{0} C}
$$

(où $\eta$ est la viscosité de la solution de concentration $C$ et $\eta_{0}$ la viscosité du solvant).

Cette quantité est, en effet, caractéristique du soluté et des interactions soluté-solvant.

Comme $\eta$ et $\eta_{0}$ sont très voisins en raison de la dilution, la détermination de $[\eta]$ exige une très grande précision sur la mesure des temps d'écoulement; les dispositifs optoélectroniques permettent d'obtenir une reproductibilité de $10^{-2} \mathrm{~s}$.

En général $\eta$ dépend également de la contrainte de cisaillement $\tau$; il en est de même de $[\eta]$ et l'étude de la variation de $[\eta]$ avec $\tau$ (comportement non newtonien) a fait l'objet de nombreux travaux [1]. Expérimentalement, on mesure $\eta_{0}$ et $\eta$ (pour plusieurs valeurs de $C$ ) à différentes valeurs de $\tau$, l'extrapolation (1) se faisant à $\tau=$ Cte. Il est donc nécessaire de disposer d'une gamme de tensions de cisaillement parfaitement reproductibles durant plusieurs jours (durée d'une expérience).

Dans le cas de viscosimètres capillaires, la méthode la plus courante pour faire varier la contrainte de cisaillement consiste à appliquer une pression extérieure réglable à l'amont du liquide en écoulement. Pour la régulation de cette pression, plusieurs solutions ont été proposées. Celles de Van Der Wyk et Meyer [2] (compression d'un gaz par une pression hydrostatique d'eau) et de Chan [3] (mesure simultanée des temps d'écoulement du solvant et de la solution) ne se prêtent guère à l'automatisation. Lohman- der [4] et Quadrat [5] utilisent des manostats à mercure équipés de contacts électriques fixes auxquels sont asservies des électrovannes; une telle installation est facile à automatiser mais le préréglage définitif des pressions en limite les possibilités.

Nous allons décrire une régulation de pression permettant d'obtenir 16 valeurs réglables et reproductibles de la contrainte de cisaillement. Le cycle des pressions a été entièrement automatisé ; en combinant cette installation avec un viscosimètre à mesure électronique des temps, l'intervention de l'opérateur est réduite au minimum.

Description du montage. - La régulation de pression a été adaptée à un viscosimètre Ostwald à mesure et impression automatique des temps type GramainLibeyre [6]. L'ensemble de l'installation est placé dans un local thermostaté au $1 / 10^{\mathrm{e}}$ de degré près.

La régulation s'effectue en deux temps. Un gaz comprimé (par exemple de l'azote) est d'abord amené à un régulateur pneumatique à came $\mathrm{RP}$. Ce régulateur permet de maintenir la pression constante à $1 \%$ près durant un temps prédéterminé par le découpage de la came. La consigne de pression affichée est légèrement supérieure (de 0,5 à $1 \mathrm{mb}$ ) à la pression d'utilisation.

La régulation fine s'effectue à l'aide de deux manomètres, à eau pour les surpressions de 0 à $270 \mathrm{mb}$, et à mercure pour le domaine de 270 à $1200 \mathrm{mb}$. Ces manomètres ont été transformés en manostats par l'adjonction des capteurs optoélectroniques $C_{i}$ pouvant coulisser le long des tubes manométriques.

Ces capteurs sont constitués par une lampe et une photodiode OAP 12 ; le passage du ménisque du liquide dans le faisceau lumineux provoque une variation du courant de photodiode et permet de comman- 


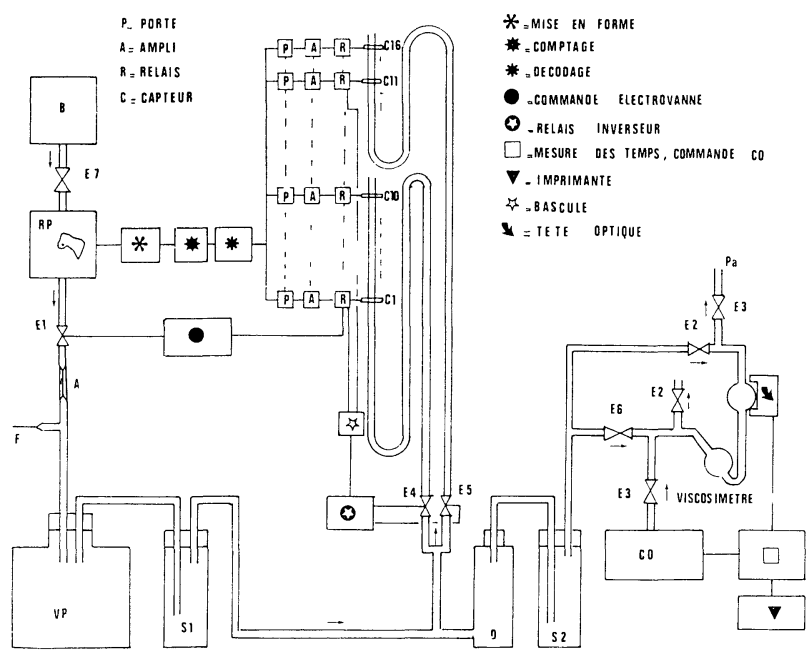

FIG. 1. - Schéma de l'appareil.

der l'ouverture (insuffisance de pression) ou la fermeture de l'électrovanne $E_{1}$. En plaçant après RP une fuite capillaire $\mathrm{F}$ par laquelle le gaz s'échappe en permanence et un étranglement réglable $\mathrm{A}$, le liquide-manométrique effectue des oscillations rapides autour de la position définie par le faisceau lumineux. L'amplitude de ces oscillations se règle grâce à $\mathrm{A}$, mais reste toujours faible en raison de la rapidité de l'asservissement de $\mathrm{E}_{1}$ et de la prérégulation due à $\mathrm{RP}$.

Le montage comporte, en outre, un réservoir de 20 litres (VP) servant de volant de pression, 2 saturateurs à solvant $S_{1}$ et $S_{2}$ et un dessicateur $\mathrm{D}$ au chlorure de calcium et au silicagel.

Automatisation des mesures. - a) MESURE sous UNE PRESSION DONNÉE. - Le viscosimètre GramainLibeyre permet la mesure et l'impression automatique des temps d'écoulement ainsi que la répétition de ces mesures ; en effet, le liquide est ramené automatiquement au niveau de mesure au moyen d'un petit compresseur $\mathrm{CO}$. Le passage du ménisque liquide au niveau inférieur de mesure du viscosimètre provoque la mise en route du compresseur, la fermeture des électrovannes $E_{2}$ et $E_{2}^{\prime}$ et l'ouverture des électrovannes $E_{3}$ et $E_{3}^{\prime}$. Le passage du liquide au niveau supérieur de mesure commande l'arrêt du compresseur, la fermeture de $\mathrm{E}_{3}$ et $\mathrm{E}_{3}^{\prime}$ et l'ouverture de $\mathrm{E}_{2}$ et $\mathrm{E}_{2}^{\prime}$.

b) Passage d'une pression a l'autre. AutomaTISATION DU CYCLE. - La consigne de pression du prérégulateur pneumatique RP est affichée sur une came tournant sous l'action d'un moteur synchrone. La came est découpée en fonction du programme envisagé, les temps étant déterminés pour permettre au moins 6 mesures du temps d'écoulement pour chaque valeur de la contrainte de cisaillement. A la fin de chaque palier de pression $\mathrm{P}_{\mathrm{i}}$, une encoche dans la came actionne un micro-contact électrique. Le signal ainsi obtenu est utilisé après comptage, décodage et mise en forme (voir annexe) pour la mise hors circuit du capteur $\mathrm{C}_{\mathrm{i}}$ et l'enclanchement du capteur $\mathrm{C}_{\mathbf{i}+1}$. La pression augmente alors progressivement dans l'installation jusqu'à atteindre la pression correspondant au niveau du capteur $\mathrm{C}_{\mathbf{i}+1}$ qui en assure alors la régulation.

Dix capteurs ont été montés sur le manostat à eau et six sur le manostat à mercure. Le changement de manomètre après la $10^{\mathrm{e}}$ pression est commandé par une bascule et un relais inverseur agissant sur les électrovannes $\mathrm{E}_{4}$ et $\mathrm{E}_{5}$. (Isolement du manomètre à eau et mise sous pression du manomètre à mercure.)

$\mathrm{La}$ pression délivrée par le compresseur $\mathrm{CO}$ est d'environ $100 \mathrm{mb}$. Aux pressions supérieures à $\mathrm{P}_{6}$, une remontée plus rapide du liquide dans le viscosimètre peut être obtenue en faisant agir par $\mathrm{E}_{6}$, la pression de gaz régulée et en mettant $\mathrm{CO}$ hors circuit; c'est également le signal dû à l'encoche de fin du $6^{\mathrm{e}}$ palier qui commande ce changement.

L'encoche de fin du palier de pression le plus élevé (16e palier) provoque l'isolement de la source de pression (vanne $\mathrm{E}_{7}$ ), la mise à la pression atmosphérique de l'installation et l'arrêt électrique complet.

Résultats. - Les performances de cet appareillage ont été mesurées au moyen des 3 types d'expériences suivants :

- Mesure des temps d'écoulement à une pression donnée, choisie arbitrairement, durant un temps assez long $\left(1^{\mathrm{er}}\right.$ test de précision).

- Mesure des temps d'écoulement à différentes pressions, dans les conditions expérimentales courantes $\left(2^{\mathrm{e}}\right.$ test de précision).

- Répétition, sur un intervalle d'une semaine, d'une mesure de temps d'écoulement sous une pression déterminée (test de fidélité).

Ces mesures ont été faites avec du toluène pur s'écoulant dans des capillaires de rayons 0,10 et $0,15 \mathrm{~mm}$, de longueur $100 \mathrm{~mm}$, à la température de $25^{\circ} \mathrm{C}$ régulée à mieux que $10^{-2}{ }^{\circ} \mathrm{C}$.

Pendant une durée de 7 heures, sous une pression de 0,037 7 bar, le temps d'écoulement moyen est $t=453,223 \mathrm{~s}$ avec un écart quadratique moyen de $0,034 \mathrm{~s}$.

Les résultats et les écarts quadratiques moyens obtenus avec les deux autres types d'essais sont représentés dans les tableaux I et II, où figurent les valeurs des pressions $P$, les temps moyens d'écoulement $t$, le rayon $R$ du capillaire, et le gradient de vitesse à la paroi $G$.

On voit que la précision relative sur la mesure des temps d'écoulement est supérieure à $2 \times 10^{-4}$ pour des pressions appliquées variant de 0,02 à 1 bar et des temps d'écoulement compris entre 10 et $900 \mathrm{~s}$. Ainsi, les performances du viscosimètre automatique ne sont pas affectées de façon notable par l'adjonction de l'installation de pression qui vient d'être décrite. La reproductibilité, durant 7 jours, des temps d'écoulement, garante de celle des contraintes de cisaillement, permet d'éliminer une cause importante d'erreurs dans les extrapolations de [ $\eta]$. Enfin, l'utilisation de capteurs mobiles permet de modifier 


\section{TABLEAU I}

Temps d'écoulement sous différentes pressions

\begin{tabular}{ccrcc}
$P_{\text {bar }}$ & \multicolumn{1}{c}{$R_{\mathrm{mm}}$} & \multicolumn{1}{c}{$t_{\mathrm{s}}$} & \multicolumn{1}{c}{$\boldsymbol{G}$} & $B / P s^{-1} \times 10^{2}$ \\
$\overline{0,0206}$ & $\overline{0,10}$ & $833,857 \pm 0,151$ & 1840 & 0,04 \\
0,0377 & 0,10 & $453,223 \pm 0,034$ & 3370 & 0,08 \\
0,0733 & 0,10 & $233,258 \pm 0,029$ & 6540 & 0,14 \\
0,4940 & 0,10 & $34,879 \pm 0,003$ & 44100 & 0,94 \\
0,8230 & 0,10 & $21,041 \pm 0,002$ & 73480 & 1,54 \\
0,0186 & 0,15 & $122,732 \pm 0,018$ & 2490 & 0,37 \\
0,0355 & 0,15 & $64,625 \pm 0,002$ & 4750 & 0,76 \\
0,0515 & 0,15 & $44,828 \pm 0,005$ & 6900 & 1,02 \\
0,0697 & 0,15 & $33,135 \pm 0,004$ & 9330 & 1,37 \\
0,1344 & 0,15 & $17,379 \pm 0,004$ & 18000 & 2,58 \\
0,1787 & 0,15 & $13,183 \pm 0,001$ & 23930 & 3,38 \\
0,2329 & 0,15 & $10,215 \pm 0,002$ & 31190 & 4,33
\end{tabular}

TABLEAU II

Répétition d'une mesure sous 0,1770 bar

$t(\mathrm{~s})$

$\begin{array}{lll}1^{\text {er }} \text { jour } & 96,530 \pm 0,005 & \\ 2^{\mathrm{e}} \text { jour } & 96,547 \pm 0,005 \\ 3^{\mathrm{e}} \text { jour } & 96,528 \pm 0,005 \\ 4^{\mathrm{e}} \text { jour } & 96,519 \pm 0,003 \\ 6^{\mathrm{e}} \text { jour } & 96,522 \pm 0,008 & \\ 7^{\mathrm{e}} \text { jour } & 96,529 \pm 0,004 & \end{array}$

facilement les valeurs des pressions en fonction des nécessités des expériences.

Limitation de l'appareillage. - Plusieurs facteurs contribuent à limiter les valeurs de la contrainte de cisaillement que l'on peut obtenir avec l'installation qui vient d'être décrite. La plupart des liaisons sont assurées par des rodages en verre et on ne peut dépasser une pression de 1,2 bar. Par ailleurs, pour garder une précision convenable sur les temps d'écoulement, nous nous limiterons à des temps supérieurs à $10 \mathrm{~s}$. Compte tenu des caractéristiques des viscosimètres, l'écoulement sera laminaire (nombre de Reynolds $<1500$ [7]) pour des liquides de viscosité supérieure à 0,4 centipoise (ce qui est le cas de la plupart des solvants). Nous avons calculé la valeur de la contrainte de cisaillement à la paroi $\tau_{\mathrm{m}}$ qu'il est possible d'atteindre pour le débit maximum $Q_{\mathrm{m}}$ (correspondant au temps d'écoulement de $10 \mathrm{~s}$ ) compte tenu de la gamme de diamètres de capillaires de précision commercialisée (à partir de $0,2 \mathrm{~mm}$ ). On a

$$
\tau=\frac{P R}{2 l}
$$

et en première approximation,

$$
Q=\frac{V}{t}=\frac{\pi P R^{4}}{8 \eta l}
$$

où $R$ et $l$ sont respectivement le rayon et la longueur du capillaire, $P$ la pression agissante, $Q$ le débit, $V$ le volume qui s'écoule pendant le temps $t$. D'où

$$
\tau=\frac{4 Q}{\pi R^{3}} \eta
$$

REVUE DE PHYSIQUe APPLIQUée. - T. 7, No 4, DÉCEMBRE 1972 et

$$
\tau_{\mathrm{m}}=\frac{4 Q_{\mathrm{m}}}{\pi R^{3}} \eta
$$

On obtient ainsi des droites de pente

$$
G_{\mathrm{m}}=\frac{4 Q_{\mathrm{m}}}{\pi R^{3}} \quad\left(Q_{\mathrm{m}}=0,1 \mathrm{~cm}^{3} \mathrm{~s}^{-1}\right)
$$

représentées sur la figure 2. Les horizontales en pointillé indiquent la limitation due à la pression de 1,2 bar.

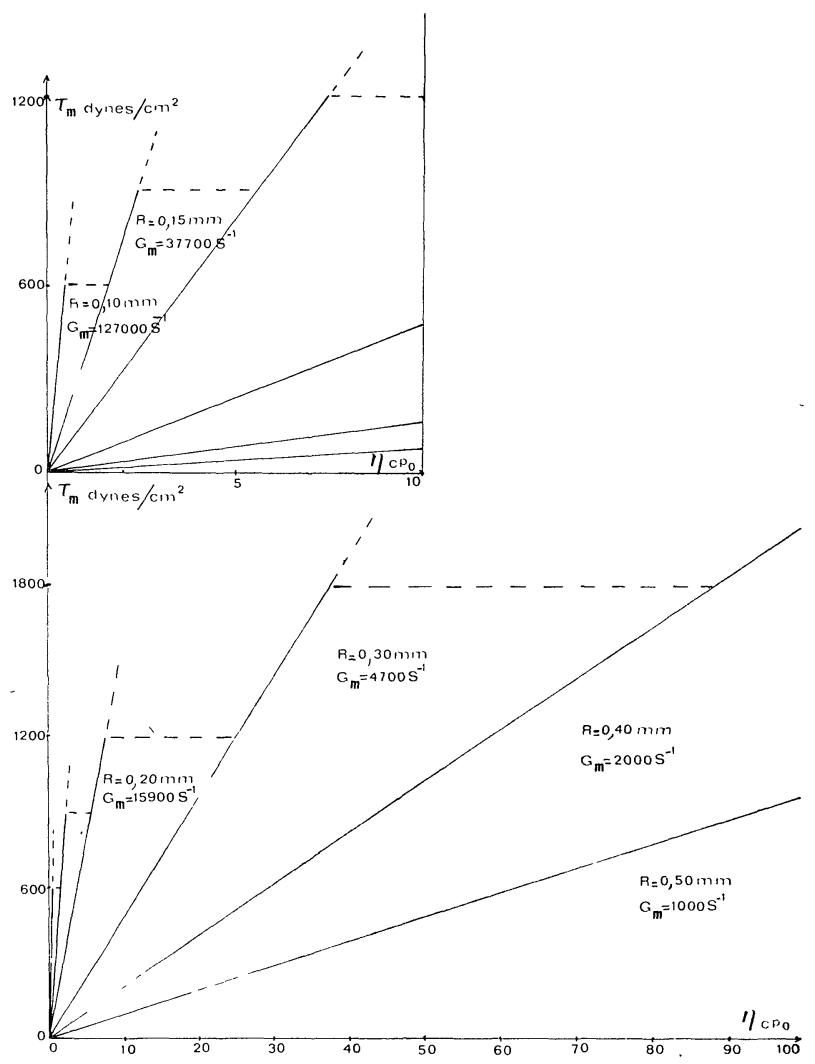

FIG. 2. - Tension de cisaillement maximum en fonction de la viscosité du solvant pour les différents diamètres de capillaires.

La vitesse du liquide atteint des valeurs élevées et le calcul de la viscosité doit alors tenir compte de la correction d'énergie cinétique.

L'expression classique

$$
\eta=\frac{\pi P R^{4} t}{8 l V}-\frac{m \rho V}{8 \pi l t}
$$

où $m$ est une constante et $\rho$ la masse spécifique du liquide, peut se mettre sous la forme

$$
\eta=A P t\left(1-\frac{B}{P t^{2}}\right)
$$

avec

$$
A=\frac{\pi R^{4}}{8 l V} \quad \text { et } \quad B=\frac{m \rho V^{2}}{\pi^{2} R^{4}} .
$$

Dans le tableau II, nous avons porté la valeur du terme de correction d'énergie cinétique $B / P t^{2}$.

$B$ est la pente de la droite représentant $P t$ en fonction de $1 / t$. Les erreurs sur $B$ sont dues, d'une part 
aux erreurs de mesure de la pression, d'autre part au fait qu'il est possible que la loi (2) ne soit qu'approchée et que $B$ dépende de la pression [8]. L'erreur sur $B$ peut jouer un rôle important dans la détermination de la viscosité spécifique $\eta_{\mathrm{sp}}=\left(\eta-\eta_{0}\right) / \eta_{0}$ aux valeurs élevées de la contrainte de cisaillement. Dans les conditions les plus défavorables, le terme $B / P t^{2}$ peut atteindre 0,1 et l'erreur $(\Delta B / B) 10 \%$. Dans ces conditions, pour une viscosité relative $\eta / \eta_{0}$ de 1,5 , l'incertitude relative sur $\eta_{\mathrm{sp}}$ due à $B$ est alors de $4 \%$. Mais cette erreur diminue avec $\eta / \eta_{0}$ et l'incertitude relative sur $[\eta]$ obtenue par extrapolation n'est que de $2 \%$, ce qui ne modifie que faiblement l'allure des courbes $[\eta]=f(\tau)$, puisque seuls les points obtenus aux valeurs extrêmes de $\tau$ sont affectés.
Conclusion. - L'appareillage décrit ci-dessus permet d'effectuer un cycle de mesures des temps d'écoulement sous 16 contraintes de cisaillement différentes sans intervention de l'opérateur. Les pressions de gaz comprimé créant ces contraintes de cisaillement peuvent être ajustées de façon continue entre 0,01 et 1,2 bar. Le dispositif de régulation assure aux pressions une stabilité et une reproductibilité déterminant une précision dans la mesure des temps d'écoulement qui est de $2 \times 10^{-4}$ avec du toluène.

Remerciements. - Monsieur Pierre Coribras, aide technique, nous a fourni une aide très précieuse. Il a réalisé l'ensemble du montage électronique et nous l'en remercions vivement.

Annexes. - Schémas de la commande de l'électrovanne $\mathrm{E}_{1}$ et du dispositif de mise en forme, comptage et décodage ( $\mathrm{P}$. Coribras).

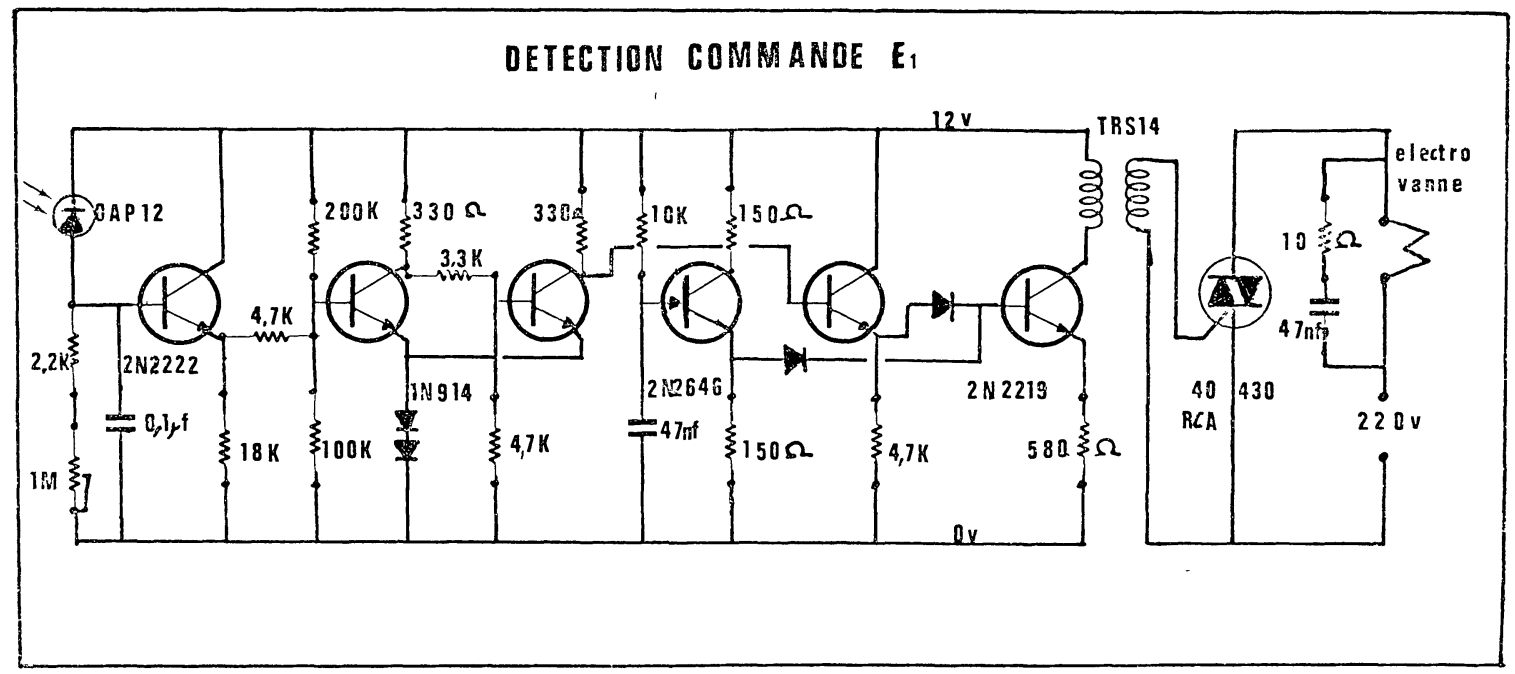

AnNexe 1. - Commande de l'électrovanne $\mathrm{E}_{1}$.
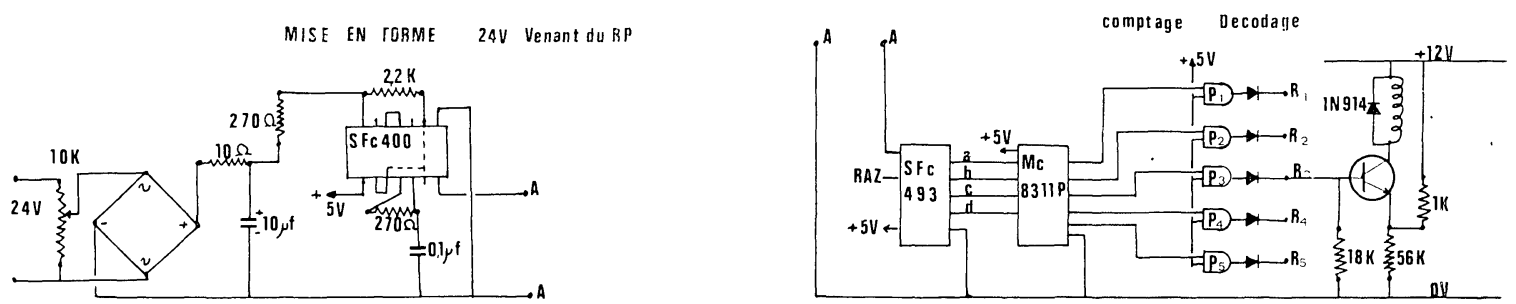

ANNEXe 2. - Dispositif de mise en forme, comptage et décodage.

\section{Bibliographie}

[1] Wolff (C.). - J. Physique, 1971, 32, C5a, 263.

[2] VAN DER WyK (A.), MeYer (K. H.). - J. Chem. Phys., $1935,32,549$

[3] Chan (F. S.). - J. Appl. Polym. Sci., 1971, 15, 1703.

[4] Claesson (S.), Lohmander (U.). - Makromoleculare Chemie, 1961, 45-46, 461.

[5] Quadrat (O.). - Chem. Listy, 1966, 60, 6, 825.
[6] Gramain (P.), Libeyre (R.). - J. Appl. Polym. Sci., $1970,14,2,383$.

[7] Lindaren (R. E.). Ark. Fys., 1959, 15, 503.

[8] Cannon (M. R.), Manning (R. E.), Bell (J. D.). - Anal. Chem., 1960, 32, 355.

Le viscosimètre Gramain-Libeyre est commercialisé par les Sociétés Fica pour l'Europe et Bausch et Lomb pour l’Amérique. 